This item was submitted to Loughborough's Research Repository by the author.

Items in Figshare are protected by copyright, with all rights reserved, unless otherwise indicated.

\title{
An ultra-low frequency magnet-tethered vibration energy harvester for self- powered sensing
}

PLEASE CITE THE PUBLISHED VERSION

https://doi.org/10.1109/Transducers50396.2021.9495714

PUBLISHER

IEEE

VERSION

AM (Accepted Manuscript)

\section{PUBLISHER STATEMENT}

Personal use of this material is permitted. Permission from IEEE must be obtained for all other uses, in any current or future media, including reprinting/republishing this material for advertising or promotional purposes, creating new collective works, for resale or redistribution to servers or lists, or reuse of any copyrighted component of this work in other works.

\section{LICENCE}

All Rights Reserved

\section{REPOSITORY RECORD}

Masabi, SN, Hailing Fu, and Stephanos Theodossiades. 2021. "An Ultra-low Frequency Magnet-tethered Vibration Energy Harvester for Self-powered Sensing”. Loughborough University. https://hdl.handle.net/2134/16681999.v1. 


\section{AN ULTRA-LOW FREQUENCY MAGNET-TETHERED VIBRATION ENERGY HARVESTER FOR SELF-POWERED SENSING \\ Sayed Nahiyan Masabi ${ }^{l}$, Hailing $\mathrm{Fu}^{1}$, and Stephanos Theodossiades ${ }^{1}$ \\ ${ }^{1}$ Wolfson School of Mechanical, Electrical and Manufacturing Engineering, Loughborough University, Leicestershire, LE11 3TU, UK}

\begin{abstract}
Wireless sensors face challenges regarding power supply and maintenance when batteries are used. To provide an effective solution, this paper presents a bistable energy harvester capable of exploiting ultra-low frequency vibration to energize low-powered devices. The energy harvester uses rotary-translational motion of a spherical rolling magnet contained inside a frame, with two tethering magnets below the rolling magnet's path to enable two stable positions. To examine the performance of the design, a prototype was fabricated and tested under varying excitation conditions and external loads. An output of $7.1 \mathrm{~mW}$ at $750 \Omega$ was effectively determined. A power management circuit is integrated with the energy harvester; the harvester can charge a $470 \mu \mathrm{F}$ capacitor to $3.4 \mathrm{~V}$ within $82 \mathrm{~s}$ at $0.7 \mathrm{~g}$ and $2 \mathrm{~Hz}$.
\end{abstract}

\section{KEYWORDS}

Vibration energy harvesting; Bi-stability; Ultra-low frequency excitation; Self-powered sensing.

\section{INTRODUCTION}

Wireless sensor networks (WSN) are widely used in various applications such as healthcare and aerospace to provide real-time distributed sensing. WSNs consist of static or portable sensor nodes for multi-hop transmission [1]. However, the current WSNs used in all sectors come with a number of limitations. For both stationary and remote sensing nodes, one of the main concerns is the limited energy in the nodes supplied by batteries and the difficulties faced while replacing them from widespread locations where they are deployed [2]. To address this problem, energy harvesters $(\mathrm{EH})$ as an alternative to using batteries have gained significant research interest. Ambient sources are used for energy harvesting, including photons, phonons, mechanical vibration, and fluid flow. Ambient vibrations from hosts where sensors are mounted can be converted into electrical energy using $\mathrm{EH}$, but the broad and low frequency vibrations create a challenge of harvesting sufficient energy to meet the required sensing demands [3]. Additionally, linear energy harvesters tend to have a narrow operating bandwidth which is a limiting factor for such devices [4].

To address these concerns, a significant number of $\mathrm{EH}$ applications have been studied, focusing on type of transduction, nonlinearity, stability, and types of motions [5-6]. Piezoelectric and electromagnetic transduction have gained popularity due to their higher power density and relative simplicity of implementation. Fu et al. presented a broadband piezoelectric EH solution in a host-parasite structure using bi-stability and frequency up conversion, generating over $1 \mathrm{~mJ}$ in $100 \mathrm{~s}$ from random excitations [7]. Abdelnaby and Arafa [8] proposed an electromagnetic $\mathrm{EH}$ capable of generating $0.14 \mathrm{~mW}$ from $5 \mathrm{~Hz}$ excitation frequency and $1.8 \mathrm{~mm}$ excitation amplitude. Other critical influences in energy harvesting using electromagnetic transductions include applications of cantilever beams [810], rotational mechanisms [11-14] and pendulum-based structures [15-17].

This paper introduces an ultra-low frequency energy harvester that utilizes rotary-translational motion of a rolling magnet, applying the theoretical model established in [18]. A prototype is developed, and its performance to exploit ultra-low vibration is explored in a comprehensive experimental study.

\section{ULTRA-LOW FREQUECY HARVESTER}

The layout of the proposed energy harvester is presented in Fig. 1. A sphere magnet is contained within a cuboid chassis where the magnet can roll along the length of the cuboid. Underneath the rolling path, two tethering magnets are placed such that the attracting forces between the rolling magnet and the tethered magnets can generate two stable positions on the rolling path.

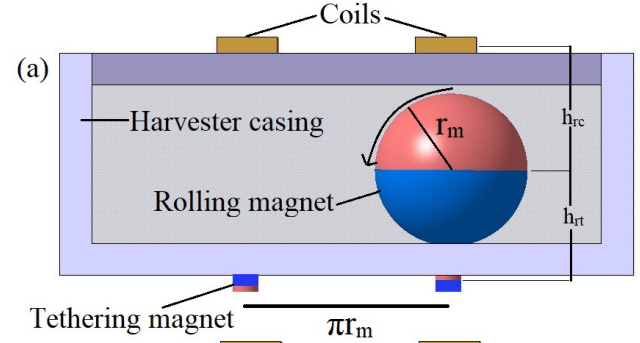

(b)
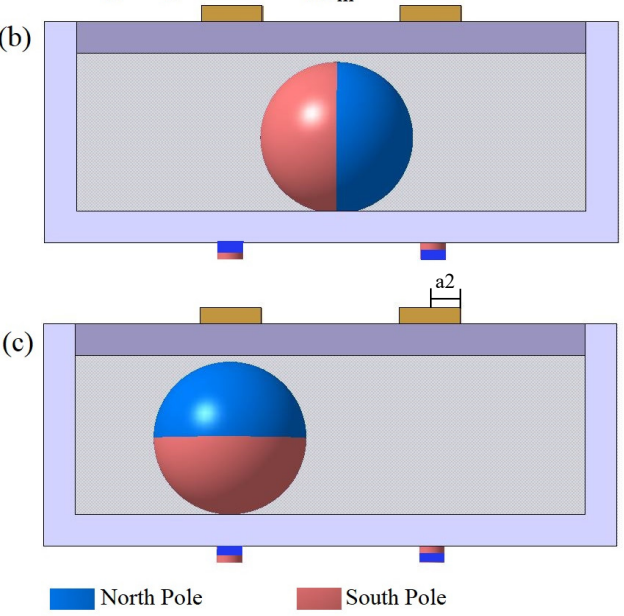

Figure 1: Schematic of the proposed ultra-low frequency energy harvester using the rotary-translation motion.

The polarity of the tethered magnets is reversed relative to each other in order to generate attractive force at both stable positions of the rolling magnet, as demonstrated in figure 1(a) and 1(c). This is because the distance between the tethered magnets is $\pi r_{m} \quad(0.5$ perimeter of the sphere) and as the rolling magnet moves from one stable position to another, its polarity reverses. 
Two stable positions enhance the energy harvesting capability of the device over a wide bandwidth. For generating electricity, two coils are fixed on top of the rolling path and directly above the tethered magnets. As the rolling magnet oscillates along the path due to excitation, magnetic flux change occurs within the coils and voltage is induced through the process of electromagnetic induction. The variation of forces applied by the tethering magnets on the rolling magnet and the generated potential energy are shown in Fig. 2, using the theoretical model developed in [18]. As shown in Fig. 2(b), the input excitation needs to be high enough to conquer the potential barrier for achieving double-well oscillations.
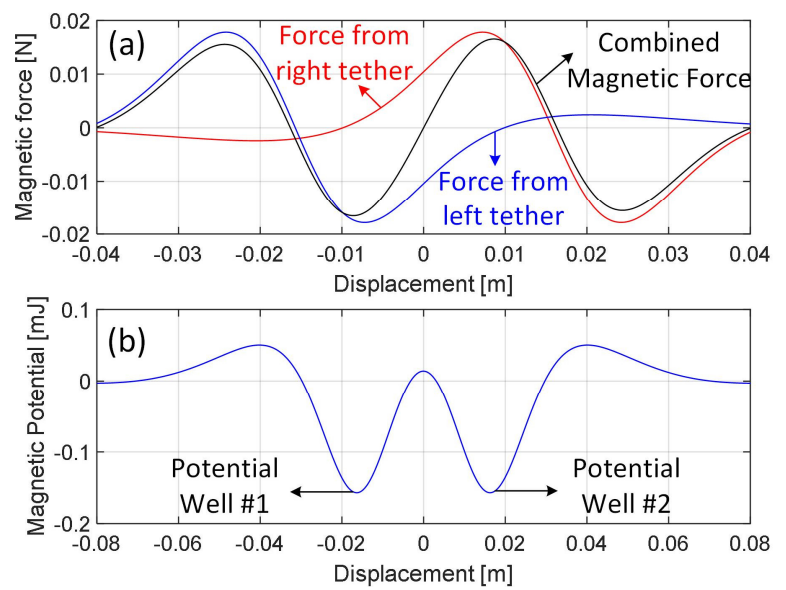

Figure 2: Magnetic force from tethering magnets (a) and the magnetic potential energy generated by the tethering magnet (b), as a function of rolling magnet displacement.

\section{EXPERIMENTAL SETUP}

The energy harvester design based on the theoretical model described in [18] was used to fabricate a prototype, as pictured in Fig. 3 to explore its performance and ability to perform under varying excitation amplitudes and frequencies. The design parameters used for this harvester are listed in Table 1.
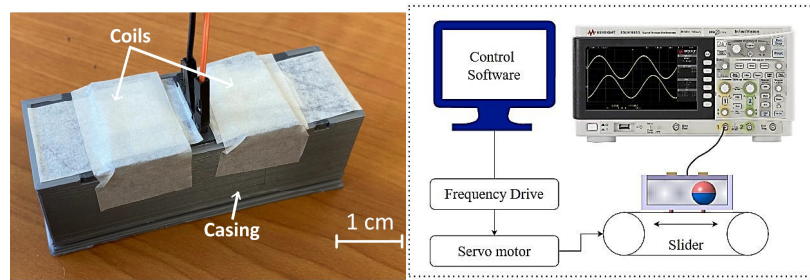

Figure 3: Harvester prototype and experimental setup

Table 1: Design parameters and material properties.

\begin{tabular}{|c|c|c|}
\hline Symbol & Description & Value \\
\hline$m$ & Rolling magnet mass & $29.4 \mathrm{~g}$ \\
\hline$\zeta$ & Mechanical damping ratio & 0.06 \\
\hline$r m$ & Rolling magnet radius & $10 \mathrm{~mm}$ \\
\hline$B r$ & Residual flux density & $1.3 \mathrm{~T}$ \\
\hline$r t$ & Tethering magnet radius & $1.5 \mathrm{~mm}$ \\
\hline$h r t$ & $\begin{array}{c}\text { Vertical gap between } \\
\text { tethering and rolling } \\
\text { magnet }\end{array}$ & $24.5 \mathrm{~mm}$ \\
\hline$h r c$ & Vertical gap between coils & $12 \mathrm{~mm}$ \\
\hline
\end{tabular}

\begin{tabular}{|c|c|c|}
\hline & and rolling magnet & \\
\hline$a 1$ & Inner radius of coils & $3 \mathrm{~mm}$ \\
\hline$a 2$ & Outer radius of coils & $12 \mathrm{~mm}$ \\
\hline$L$ & Coil inductance & $16.8 \mathrm{mH}$ \\
\hline$R c$ & Coil resistance & $388 \Omega$ \\
\hline
\end{tabular}

In order to excite the rolling magnet in a repeatable manner, the energy harvester was mounted on a conveyer belt powered by a servomotor (Kollmorgen EN60034), which was controlled by a variable frequency drive. The generated output voltage from the harvester was rectified using a full-bridge rectifier and then controlled using a power management module (BQ25505EVM) intended for low-power applications. The module contains a boost converter which could efficiently maintain a constant DC output voltage, used for charging energy storage elements.

\section{RESULTS AND DISCUSSION}

Using the experimental setup, the performance of the energy harvester was investigated and discussed. In order to understand the dynamics of the energy harvester, it was tested under a set excitation condition of $0.7 \mathrm{~g}$ amplitude and $2 \mathrm{~Hz}$ frequency. With these chosen values, the output voltage against time was recorded. Figure 4 shows the output characteristics of the device.

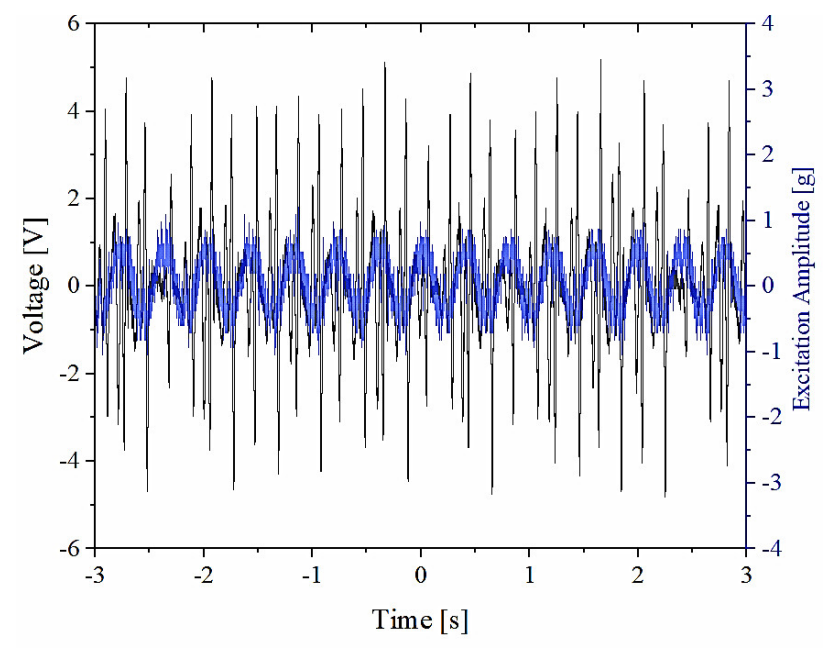

Figure 4: Open-circuit output voltage (black) and input excitation acceleration at $0.7 \mathrm{~g}$ and $2 \mathrm{~Hz}$ vibrations (blue).

The harvester was able to operate in double-well oscillatory motion, since the excitation is high enough for the rolling magnet to conquer the potential barrier created by the attracting forces of the tethering magnets. From Fig. 4, it is also apparent that for each oscillation of the rolling magnet, about 3 voltage cycles could be observed, showing that the output voltage frequency was multiplied by 3 times for each full cycle of motion. This ratio is dependent on the electromagnetic coupling factor and can be varied by changing the vertical gap between the coils and the magnet or the number of coil turns [18].

\section{Excitation Conditions}

Since it is important for the harvester to scavenge energy from vibration sources over a broad bandwidth of excitations, the output performance of the device was 
tested for incrementally increasing ultra-low values for excitation amplitudes and frequencies.

Initially, the harvester was tested under fixed vibration frequency of $2 \mathrm{~Hz}$, with varying excitations between $0.1 \mathrm{~g}$ and $1.0 \mathrm{~g}$ to record the output voltage characteristics and observe the effect of the excitation parameters at this range. Figure 5(a) presents the time history for the full excitation amplitude range at $2 \mathrm{~Hz}$. Between $0.1 \mathrm{~g}-0.3 \mathrm{~g}$, the harvester operated with singlewell oscillations, as evidently from the graph, since the output voltage is relatively lower at this scale. At $0.4 \mathrm{~g}$, the excitation is strong enough to start overcoming the potential barrier and sporadic double-well oscillations are noticeable. At excitations greater than $0.4 \mathrm{~g}$, more frequent double-well motions are noticed as gradually consistent peak-to-peak values of higher output voltage are recorded.
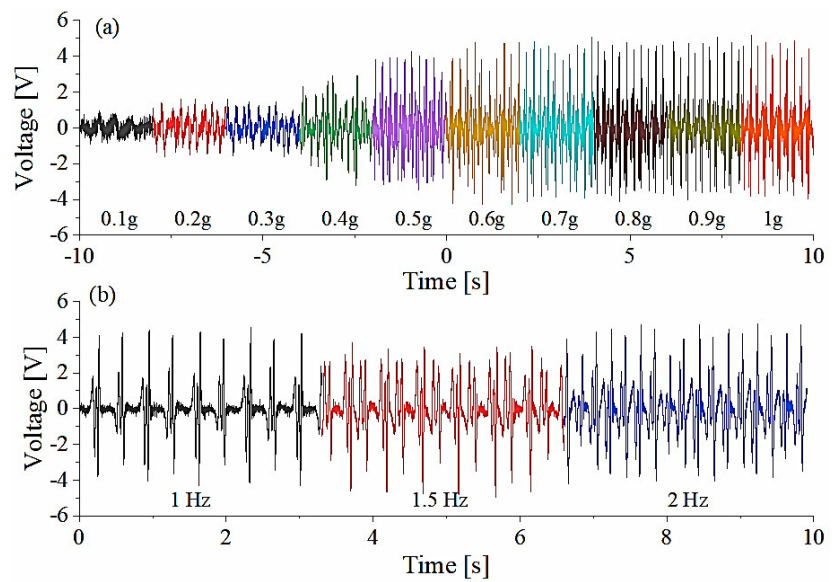

Figure 5: Output voltages at different excitations: (a) $0.1 \mathrm{~g}$ - $1.0 \mathrm{~g}$ at $2 \mathrm{~Hz}$; (b) $1 \mathrm{~Hz}-2 \mathrm{~Hz}$ at $0.7 \mathrm{~g}$ amplitude

The impact on the output voltage by changing the excitation frequency was examined at a fixed excitation amplitude (0.7g). Fig. 5(b) illustrates that with increasing frequency, double-well oscillations were consistently achieved as greater density of high peak voltage could be observed at higher excitation frequencies. These results show that the energy harvester can operate effectively at low frequencies.

\section{Impedance Matching and Maximum Power}

With the appropriate excitation conditions derived above, the output voltage and power characteristics of the device were studied. A range of external load resistance was applied to the energy harvesting circuit to examine its power supplying ability under a fixed vibration condition. The values of the loads were changed incrementally between $100 \Omega$ and $1.5 \mathrm{k} \Omega$ and the impact on the rectified output voltage is shown in Fig. 6. The output power curve was also derived as presented in Fig. 6.

The output voltage trend approximately resembles a logarithmic growth as the resistance was increased, but the power curve changed in a distinct way such that it reached peak value of $7.1 \mathrm{~mW}$ when an external load resistance of $750 \Omega$ was applied. This is the characteristic of the energy harvester circuit since it has a source impedance and optimum power transfer is obtained when external load impedance matches source impedance, as established by the maximum power transfer theorem [19]. Therefore, an output load of $750 \Omega$ results in optimal power transfer.

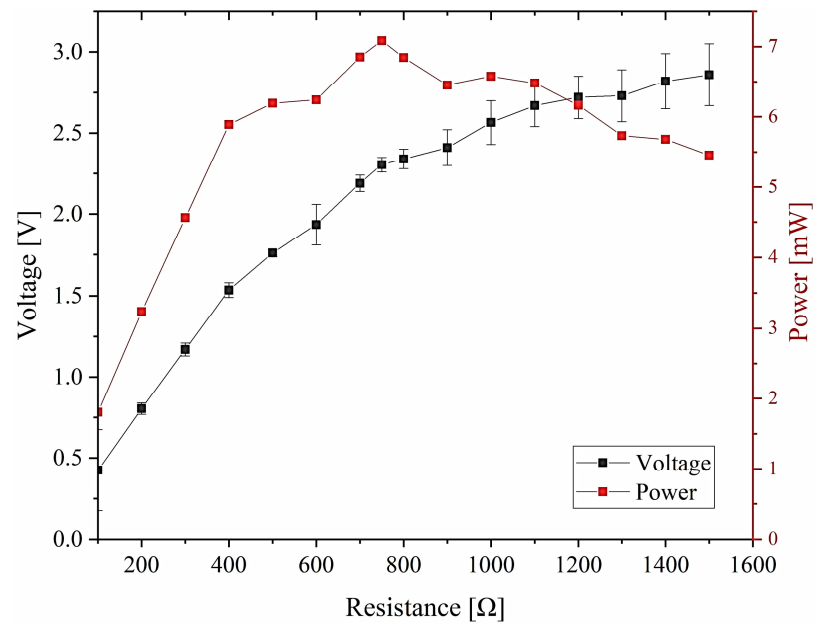

Figure 6: Impedance matching curve at $0.7 \mathrm{~g}$ and $2 \mathrm{~Hz}$ excitation conditions (rectified peak output voltage).

\section{Power Management}

The generated pulses from the energy harvester had relatively low amplitudes within the range considered, hence simple tasks such as voltage rectification could cause energy loss. In order to improve the output efficiency, the BQ25505 power management board was employed for energy storage without compromising efficiency. The board contained a boost converter which was able to keep constant DC voltage output and it could be used to charge storage units, such as capacitors.

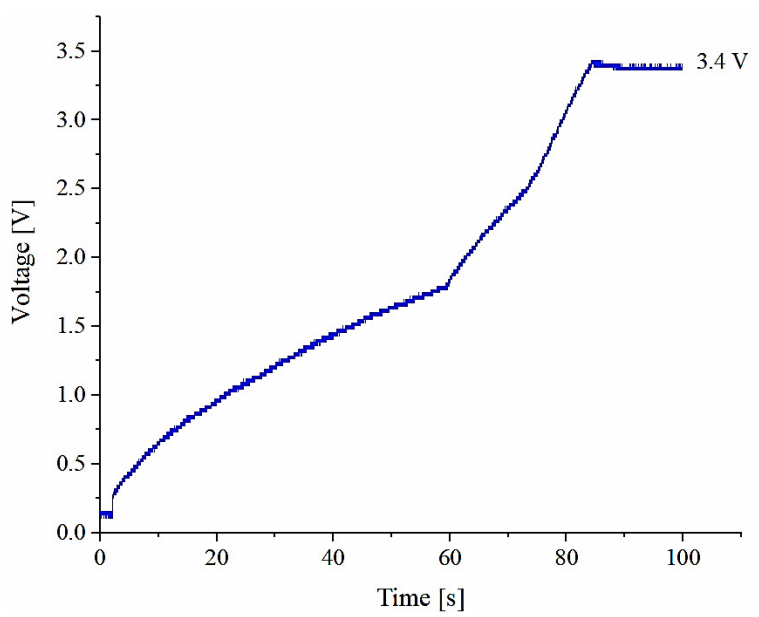

Figure 7: Capacitor charging curve from the power management output at $0.7 \mathrm{~g}$ and $2 \mathrm{~Hz}$ excitation.

A $470 \mu \mathrm{F}$ capacitor was used to demonstrate the performance of the harvester for energy storage targets. Fig. 7 presents the stated capacitor's charging curve from a discharged to fully charged state, showing a charging time of $82 \mathrm{~s}$ to reach a stable $3.40 \mathrm{~V}$. The capacitor was able to store $2.72 \mathrm{~mJ}$ of energy after $82 \mathrm{~s}$.

The power management unit functioned in a way that at lower inputs from the energy harvester, the conversion efficiency was generally low, and as the voltage reached a certain $1.8 \mathrm{~V}$, the efficiency was significantly raised for 
quicker charging of the capacitor.

\section{CONCLUSIONS}

In conclusion, an ultra-low frequency harvester based on rotary-translational motion of a rolling magnet has been presented in this paper. Attractive forces from two tethering magnets under the rolling path were used to achieve bi-stability of the rolling magnet. Depending on the level of excitation amplitude, the harvester performed in single-well or double-well oscillations. At $2 \mathrm{~Hz}$, excitation amplitudes higher than $0.4 \mathrm{~g}$ resulted in doublewell motions and higher output voltage peaks. Impedance matching results show that a maximum power output of $7.1 \mathrm{~mW}$ could be obtained with $750 \Omega$ external load at 2 $\mathrm{Hz}$ and $0.7 \mathrm{~g}$. Using a power management chip, the output from the harvester was capable of effectively charging a capacitor to $3.4 \mathrm{~V}$ within 82 seconds. This work provides a method in effectively harnessing low frequency vibrations (such as human motion or wind turbine blades) for self-powered sensing applications.

\section{ACKNOWLEDGEMENTS}

The authors would like to thank the Wolfson School of Mechanical, Electrical and Manufacturing Engineering, Loughborough University for funding this research.

\section{REFERENCES}

[1] Y. Luo, Y. Duan, W. Li and G. Fortino, "Workshop networks integration using mobile intelligence in smart factories," in IEEE Communications Magazine, vol. 56, no. 2, 2018.

[2] Y. Duan, W. Li, P. Pace and G. Fortino, "Software Defined Wireless Sensor Networks: A Review," in 2018 IEEE 22nd International Conference on Computer Supported Cooperative Work in Design ((CSCWD)), Nanjing, China, 2018.

[3] H. Li, C. Tian and Z. D. Deng, "Energy harvesting from low frequency applications using piezoelectric materials," Applied Physics Reviews, vol. 1, no. 4, p. 041301, 2014.

[4] A. Porcar-Climent and N. Jackson, "Rolling Mass for Wide bandwidth Vibration Energy Harvesting," in 2019 19th International Conference on Micro and Nanotechnology for Power Generation and Energy Conversion Applications (PowerMEMS), 2019.

[5] X. Zhang, M. Zuo, W. Yang and X. Wan, "A TriStable Piezoelectric Vibration Energy Harvester for Composite Shape Beam," Sensors, vol. 20, no. 5, p. 1370, 2020.

[6] C. Trigona, F. Maiorca, B. Ando and S. Baglio, "Tristable behavior in mechanical oscillators to improve the performance of vibration energy harvesters," in 2013 Transducers \& Eurosensors XXVII: The 17th International Conference on Solid-State Sensors, Actuators and Microsystems, 2013

[7] H. Fu, Z. S. Khodaei and M. F. Aliabadi, "Broadband Energy Harvesting Using Bi-Stability and Frequency Up-Conversion for Self-Powered Sensing in Internet of Things," in 2019 20th International Conference on Solid-State Sensors, Actuators and Microsystems \& Eurosensors XXXIII (TRANSDUCERS \&
EUROSENSORS XXXIII), 2019.

[8] M. A. M. Abdelnaby, "A bistable electromagnetic energy harvester for low-frequency, low-amplitude excitation," Journal of the Brazilian Society of Mechanical Sciences and Engineering, vol. 42, no. 520, 2020.

[9] F. M. Foong, C. K. Thein, B. L. Ooi and D. Yurchenko, "Increased power output of an electromagnetic vibration energy harvester through anti-phase resonance," Mechanical Systems and Signal Processing, vol. 116, pp. 129-145, 2019

[10] M. Jagiela and M. Kulik, "Effects of complex motion in a new cantilever-beam nonlinear electromagnetic vibration energy harvester," in 2017 18th International Symposium on Electromagnetic Fields in Mechatronics, Electrical and Electronic Engineering (ISEF) Book of Abstracts, Lodz, 2017.

[11]D. Dinulovic, M. Brooks, M. Huag and T. Petrovic, "Rotational Electromagnetic Energy Harvesting System," Physics Procedia, vol. 75, pp. 1244-1251, 2015.

[12] B. E. Gunn, S. Theodossiades and S. J. Rothberg, “A Nonlinear Concept of Electromagnetic Energy Harvester for Rotational Applications," Journal of Vibration and Acoustics, vol. 141, no. 3, p. 031005 , 2019.

[13] D. Dinulovic, M. Shousha, M. Brooks, M. Huang and T. Petrovic, "Portable rotational electromagnetic energy harvester with a maximum energy tracking power management system," in 2017 International Symposium on Power Electronics (Ee), Novi Sad, 2017.

[14] M. Cai, J. Wang and W. Liao, "Self-powered smart watch and wristband enabled by embedded generator," Applied Energy, vol. 263, p. 114682, 2020.

[15]B. Lee and G. Chung, "Design and analysis of a pendulum-based electromagnetic energy harvester using anti-phase motion," IET Renewable Power Generation, vol. 10, no. 10, pp. 1625-1630, 2016.

[16]D. Castagnetti, "A simply tunable electromagnetic pendulum energy harvester," Meccanica, vol. 54, pp. 749-760, 2019.

[17] H. Wang and J. Tang, "Electromagnetic energy harvesting from a dual-mass pendulum oscillator," in Active and Passive Smart Structures and Integrated Systems 2016, Las Vegas, 2016.

[18] H. Fu, S. Theodossiades, B. Gunn, I. Abdallah and E. Chatzi, "Ultra-low frequency energy harvesting using bi-stability and rotary-translational motion in a magnet-tethered oscillator," Nonlinear Dynamics, vol. 101(4), pp. 2131-2143, 2020.

[19] M. N. Abdallah, T. K. Sarkar and M. Salazar-Palma, "Maximum power transfer versus efficiency," 2016 IEEE International Symposium on Antennas and Propagation (APSURSI), Fajardo, PR, USA, 2016, pp. 183-184, 2016.

\section{CONTACT}

*H. Fu, tel: +441509 222514; h.fu@lboro.ac.uk 